\title{
Advances in Exercise, Physical Activity, and Diabetes Mellitus
}

\author{
Aoibhe M. Pasieka ${ }^{1}$ and Michael C. Riddell ${ }^{1}$
}

Introduction

TN 2015/2016, A NUMBER OF PAPERS were published on the challenges of exercise management for patients living with diabetes. A big focus was on testing how the artificial pancreas might function during exercise, with or without activity announcements and the addition of glucagon. Moreover, several papers revealed barriers to exercise participation for people living with type 1 or type 2 diabetes. A few papers demonstrated that many of the tools for the preservation of glucose control during and after exercise in type 1 diabetes are not being used. This year, we selected 10 papers to highlight the field of exercise and diabetes, with an emphasis on "technology" rather than on the possible mechanisms for exercise action. Our initial search was restricted to human studies and primarily on studies in which patients with diabetes severed as subject participants. We screened over 150 papers on the topic that were found on Pubmed and other common search engines published between July 1, 2015 and June 30, 2016. The following 10 papers, we think, represent some of the highlights.

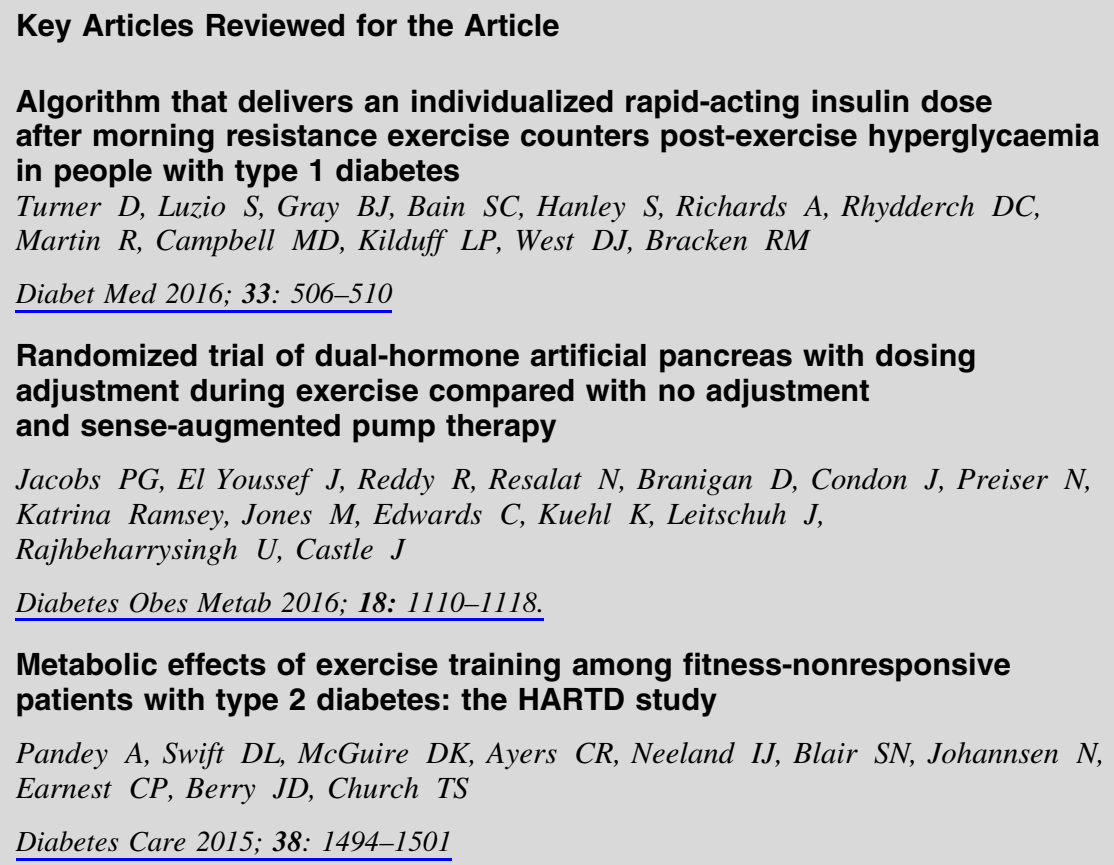

${ }^{1}$ School of Kinesiology and Health Science, York University, Toronto, ON, Canada. 
Insulin-based strategies to prevent hypoglycaemia during and after exercise in adult patients with type 1 diabetes on pump therapy: the DIABRASPORT randomized study

Franc S, Daoudi A, Pochat A, Petit MH, Randazzo C, Petit C, Martine Duclos M, Penfornis A, Pussard E, Not D, Heyman E, Koukoui F, Simon S, Charpentier G

Diabetes Obes Metab 2015; 17: 1150-1157

Impact of physical activity on glycemic control and prevalence of cardiovascular risk factors in adults with type 1 diabetes: a cross-sectional multicenter study of 18,028 patients

Bohn B, Herbst A, Pfeifer M, Krakow D, Zimny S, Kopp F, Melmer A, Steinacker JM, and Holl RW, for the DPV Initiative

Diabetes Care 2015; 38: 1536-1543

Do youth with type 1 diabetes exercise safely? A focus on patient practices and glycemic outcomes

Roberts AJ, Yi-Frazier JP, Aitken KE, Mitrovich CA, Pascual MF, Taplin CE

Pediatr Diabetes 2016; Jul 6. [Epub ahead of print] DOI 10.1111/pedi.12402

General practitioners' barriers to prescribe physical activity: the dark side of the cluster effects on the physical activity of their type 2 diabetes patients

Lanhers C, Duclos M, Guttmann A, Coudeyre E, Pereira B, Ouchchane L

PLoS One 2015; 10: e0140429.

Effects of high-intensity interval exercise versus moderate continuous exercise on glucose homeostasis and hormone response in patients with type 1 diabetes mellitus using novel ultra-long-acting insulin

Moser O, Tschakert G, Mueller A, Groeschl W, Pieber T, Obermayer-Pietsch B, Koehler G, Hofmann $P$

PLoS One 2015; 10: e0136489

Classification of physical activity: information to artificial pancreas control systems in real time

Turksoy K, Paulino TM, Zaharieva DP, Yavelberg L, Jamnik V, Riddell MC, Cinar A

J Diabetes Sci Technol 2015; 9: 1200-1207

One week of bed rest leads to substantial muscle atrophy and induces whole-body insulin resistance in the absence of skeletal muscle lipid accumulation

Dirks ML, Wall BT, van de Valk B, Holloway TM, Holloway GP, Chabowski A, Goossens GH, van Loon LJC

Diabetes 2016; 65: 2862-2875.

Algorithm that delivers an individualized rapid-acting insulin dose after morning resistance exercise counters post-exercise hyperglycaemia in people with type 1 diabetes

Turner $D^{1,2}$, Luzio $S^{2,3}$, Gray $B J^{1,2}$, Bain $S C^{2,3}$, Hanley $S^{1}$, Richards $A^{3}$, Rhydderch $D C^{3}$, Martin $R^{4}$, Campbell $M D^{5}$, Kilduff $L P^{I}$, West DJ ${ }^{5}$, Bracken RM ${ }^{1,2}$

${ }^{1}$ Applied Sports, Technology, Exercise and Medicine Research Centre, College of Engineering, Swansea University, Singleton Park, Swansea, UK; ${ }^{2}$ Diabetes Research Group, College of Medicine, Swansea University, Singleton Park, Swansea, UK; ${ }^{3}$ Abertawe Bro Morgannwg University Health Board, Singleton Hospital, Swansea, UK; ${ }^{4}$ Cwm Taf University Health Board, Merthyr Tydfil, UK; ${ }^{5}$ Department of Sport, Exercise and Rehabilitation, Faculty of Health and Life Sciences, Northumbria University, Newcastleupon-Tyne, UK

\section{Diabet Med 2016; 33: 506-510}

\section{Background}

In type 1 diabetes, weight training and other forms of anaerobic exercise can acutely increase blood glucose levels. How to manage postexercise hyperglycemia safely and effectively in this patient population is currently unclear. The goal of this study was to test an insulin dose adjustment algorithm that delivers an individualized dose of rapid-acting insulin analog after morning resistance exercise to counter postexercise hyperglycemia in individuals with type 1 diabetes. 


\section{Methods}

Eight people with type 1 diabetes ( 6 men; 2 women, all on multiple daily insulin injections (MDI)), in fair glycemic control $(8.7 \% \pm 1.1 \%$, mean \pm standard error $)$ attended two morning laboratory sessions in a fasted state, having taken their usual basal insulin the previous evening. They performed a resistance exercise session comprising of six exercises for two sets of 10 repetitions at $60 \%$ of the maximum amount of force that was generated in one maximal contraction. In a counterbalanced order, the subjects were administered either an individualized dose of rapid-acting insulin ( $\sim 2 \pm 1$ units, range 0 to 4 units) based on a modification of their own hyperglycemia correction factor (reduced by $50 \%$ ) or were not administered insulin in recovery. Venous blood glucose concentrations were measured up until $125 \mathrm{~min}$ following resistance exercise.

\section{Results}

Pre-exercise blood glucose averaged 11.2 \pm 1.2 in both sessions. Participants had a rise in blood glucose in both sessions (insulin session increased to $13.0 \pm 1.6$ vs. no-insulin session increasing to $12.7 \pm 1.5 \mathrm{mmol} / \mathrm{L} ; \quad P=0.834)$. The decline in blood glucose concentration between the peak glucose and 125 min mark after exercise was greater in the insulin administered session than in the no-insulin session $(3.3 \pm 1.0$ vs. $1.3 \pm 0.4 \mathrm{mmol} / \mathrm{L}: P=0.015)$. There were no episodes of hypoglycaemia during that same time frame in either session.

\section{Conclusions}

These authors propose that the administration of rapidacting insulin according to an individualized algorithm reduces hyperglycemia after morning resistance exercise without causing hypoglycaemia in the $2 \mathrm{~h}$ postexercise window in people with type 1 diabetes.

\section{Comment}

A large percentage of patients with type 1 diabetes have postexercise hyperglycemia following intense exercise (anaerobic and intense aerobic), likely because of sustained increases in hepatic glucose production that exceeds glucose uptake into muscle that is now finished contracting $(1,2)$. This phenomenon may be more common in patients on MDI than on continuous subcutaneous insulin infusion (CSII) (3), although it can happen in any patient who does intense exercise, particularly if there is heightened competition stress (4). High blood sugars in recovery can be prolonged (5) and can be frustrating since it may promote dehydration and compromise overall A1c levels if it is a frequent occurrence. Some athletes complain that hyperglycemia after exercise makes them feel sluggish and may compromise their capacity to recover from the activity and may wish to bolus insulin to counter these effects. However, bolusing insulin after exercise is a worry since it may increase risk for late-onset hypoglycaemia, another frustrating side effect of exercise. Our colleagues from the United
Kingdom show here that a conservative insulin dose post exercise [i.e., $50 \%$ of the typical "correction factor," based on the total daily insulin dose algorithm calculation, as described by Davidson et al. (6)] is a safe and effective way to gradually bring glucose levels into control after exercise if hyperglycemia develops. The main limitation of this pilot study is that other insulin dose administration protocols were not tested (e.g., 100\% correction, bolus before the exercise starts) and the subjects were not followed all that long in recovery (just 120 minutes).

\section{Randomized trial of dual-hormone artificial pancreas with dosing adjustment during exercise compared with no adjustment and sense- augmented pump therapy}

Jacobs $P G^{l}$, El Youssef $J^{2}$, Reddy $R^{l}$, Resalat $N^{l}$, Branigan $D^{2}$, Condon $J^{1}$, Preiser $N^{1}$, Ramsey $K^{3}$, Jones $M^{2}$, Edwards $C^{2}$, Kuehl $K^{4}$, Leitschuh $J^{1}$, Rajhbeharrysingh $U^{1}$, Castle $J^{2}$

${ }^{1}$ Department of Biomedical Engineering, Oregon Health and Science University, Portland, OR; ${ }^{2}$ Department of Medicine, Division of Endocrinology, Harold Schnitzer Diabetes Health Center, Oregon Health and Science University, Portland, OR; ${ }^{3}$ Oregon Clinical and Translational Research Institute Biostatistics \& Design Program, Oregon Health \& Science University, Portland, OR; Department of Medicine, Division of Health Promotion and Sports Medicine, Human Performance Laboratory, ${ }^{4}$ Department of Medicine, Division of Health Promotion and Sports Medicine, Human Performance Laboratory, Oregon Health and Science University, Portland, OR

Diabetes Obes Metab 2016; 18:1110-1118.

\section{Background}

Individuals with type 1 diabetes (T1D) have impaired insulin secretion and dysfunctional glucagon secretion, making them particularly susceptible to hyper- and hypoglycemia. It is generally held that artificial pancreas (AP) systems will help mitigate risk of dysglycaemia. In this system, hormone delivery is determined using an algorithm, however, with exercise, insulin sensitivity is significantly altered and adjustments are likely required. This study aimed to determine the optimal adjustments of insulin and glucagon dosing with exercise to prevent exercise induced hypoglycemia.

\section{Methods}

This study was a randomized crossover controlled study comparing dual hormone AP with adjustments (APX), versus dual hormone AP without adjustments (APN) and sensor augmented pump (SAP). Twenty-one T1D patients ages 18 to 45 stayed in a hospital overnight and had standardized meals. Postbreakfast, exercise consisted of running at $60 \%$ of HR max for 45 min on a motorized treadmill. The exercising dosing algorithm reduced insulin and increased glucagon during and postexercise for $1.5 \mathrm{~h}$. APN 
was unadjusted for exercise and SAP manually managed hormone delivery by patients.

\section{Results}

Despite similar baseline values, APX promoted significantly less time in hypoglycaemia postexercise compared to APN $(0.3 \%$ APX vs. $3.1 \%$ APN, $P=0.001)$. The APN trial had a reduced percent of time spent in hyperglycemia postexercise than both APX and SAP, although these differences did not reach significance. In the overnight period APN spent significantly less time spent in hyperglycemia than APX (8.7\% vs. $17.2 \%, P=0.04)$.

\section{Conclusions}

This study highlights the importance of adjusting insulin and glucagon doses at the onset of exercise to limit hypoglycaemia. APX performs similarly to SAP when insulin was adjusted prior to exercise.

\section{Comment}

With T1D, the body's ability to produce insulin is lost and glucagon production becomes dysregulated. As such, maintaining euglycaemia during exercise requires a lot of monitoring, manual adjustments to insulin delivery and carbohydrate snacking. For this reason, the development of the dual hormone AP for the daily monitoring and regulation of blood glucose levels is a novel and exciting branch of research for improving glycemic control in individuals with T1D. In a state of exercise, the rate of change in glycemia occurs more rapidly and in order to counteract this, the dual hormone AP system must be able to measure exercise and possibly make adjustments to both hormones. This study found that when AP was adjusted at the onset of exercise, exercise-associated hypoglycaemia was significantly reduced when compared to nonadjustment AP. SAP also resulted in relatively good glucose control, but a limit to its use is that it requires a lot more manual adjustment, which is a burden and not always feasible, especially with long, sustained exercises. Although this study was beneficial in manipulating the AP to reduce the incidences of hypoglycemia, it did not detect the onset of exercise automatically. Future research from the same group is currently aiming to incorporate an exercise detection component into the AP-algorithm by using accelerometers and heart rate inputs (7) in order to advance the field towards utilizing this technology for day-to-day use.

\section{Metabolic effects of exercise training among fitness-nonresponsive patients with type 2 diabetes: the HART-D study}

Pandey $A^{1}$, Swift $D L^{2}$, McGuire $D K^{1,3}$, Ayers $C R^{3}$, Neeland $\mathrm{IJ}^{1}$, Blair $\mathrm{SN}^{4}$, Johannsen $\mathrm{N}^{5}$, Earnest $\mathrm{CP}^{6}$, Berry $J D^{1,3}$, Church $T^{7}$

${ }^{I}$ Division of Cardiology, Department of Internal Medicine, University of Texas Southwestern Medical Center, Dallas, $T X ;{ }^{2}$ Department of Kinesiology, East Carolina University,
Greenville, NC; ${ }^{3}$ Department of Clinical Sciences, University of Texas Southwestern Medical Center, Dallas, TX; ${ }^{4}$ Department of Exercise Science, Department of Epidemiology and Biostatistics, Arnold School of Public Health, University of South Carolina, Columbia, SC; ${ }^{5}$ School of Kinesiology, College of Human Sciences \& Education, Louisiana State University, Baton Rouge, LA; ${ }^{6}$ Department for Health \& Kinesiology, Texas A\&M University, College Station, TX; ${ }^{7}$ Preventive Medicine Laboratory, Pennington Biomedical Research Center, Baton Rouge, LA

Diabetes Care 2015; 38: 1494-1501

\section{Background}

Physical activity is closely associated with cardiorespiratory fitness (CRF), yet some individuals are nonresponsive to exercise and do not experience these expected improvements (termed "nonresponders"). This study examined how type 2 diabetes (T2D) patients respond to exercise training, focusing on changes in CRF, hemoglobinA1c (HbA1c) levels and other metabolic parameters.

\section{Methods}

This study analyzed data from patients who participated in the Heath Benefits of Aerobic and Resistance Training in Diabetes (HART-D) study. There were 202 participants assigned to supervised training protocols for 9 months. The groups consisted of a nonexercise control group that received stretching and relaxation classes, an aerobic-training group (3 to 5 days/week, $50 \%$ to $80 \%$ of maximal aerobic capacity $\left[\mathrm{VO}_{2} \max \right]$ ), a resistance-training group (3 days/week, 10 to 12 reps of each activity), and a combination-training group receiving both resistance and aerobic training. Greater than $5 \%$ increase in peak oxygen uptake was considered a meaningful improvement in $\mathrm{CRF}$, otherwise, they were considered nonresponders.

\section{Results}

Only $36.6 \%$ of exercisers were considered CRF responsive (improvements in $\mathrm{VO}_{2} \max <5 \%$ ). Both responders and nonresponders in the exercise groups had significantly decreased in $\mathrm{HbA} 1 \mathrm{c}$ from baseline $(-0.26 \%$ [95\% confidence interval (CI) -0.5 to -0.01$],-0.26 \%$ [95\% CI $-0 / 45$ to $-0.08]$ ). Additionally, both responders and nonresponders had reduced waist circumference (WC) and percent body fat $(-2.6 \mathrm{~cm}[95 \% \mathrm{CI}-3.7$ to -1.5$]$ vs. $-1.8 \mathrm{~cm}[95 \% \mathrm{CI}-2.6$ to -1.0$])$, (\% body fat $-1.07 \%$ [95\% CI -1.5 to -0.62 ] vs $-0.75 \%$ [95\% CI -1.09 to -0.41$]$ ). Lastly, both responsive and nonresponsive exercisers tended to report a reduced use of diabetes-related oral agents compared to controls ( $36 \%$ vs. 20.5\%; $P=0.08$ ).

\section{Conclusions}

Exercise is associated with significant improvements in glycemic control, reductions in percent body fat/waist circumference, independent of the cardiorespiratory response to training. Based on this study, it is recommended that exercise be prescribed to T2D patients to decrease their risk of diabetes related comorbidities and improve their glucose control, with little regard to if the exercise makes their cardiorespiratory system more fit or not. 


\section{Comment}

Metabolic parameters such as cardiorespiratory fitness and waist circumference are inversely associated with insulin resistance $(8,9,10)$. Regular exercise is known to improve these factors, but for some, termed "nonresponsive" individuals, improvements in these factors are not seen, even with supervised and regular exercise. This is the first study to compare the effects of chronic moderate-intensity activity on T2D patients in responders versus nonresponders.

This was a large study $(n=202)$ with long-term supervised training interventions. It was found that in their population of only T2D adults, a surprising $63 \%$ of the subjects were considered fitness nonresponsive, a value that was quite high, especially when compared to the general population. In a recent study, only $17.6 \%$ of obese and otherwise healthy subjects were considered nonresponders after undergoing an exercise program with frequent low intensity exercise sessions (11). The reason for the increased prevalence of nonresponders with T2D requires further investigation. The lack of improvement in fitness may be discouraging for individuals and may influence exercise compliance and commitment to an exercise program. However, despite no clinical improvements in CRF, exercise is independently associated with improved glucose control, reduced insulin resistance and less body fat, thus validating that exercise is still a beneficial therapy for diabetes treatment (8).

\section{Insulin-based strategies to prevent hypoglycaemia during and after exercise in adult patients with type 1 diabetes on pump therapy: the DIABRASPORT randomized study}

Franc $S^{1,2}$, Daoudi $A^{l}$, Pochat $A^{l}$, Petit $M^{3} H^{l}$, Randazzo $C^{1}$, Petit $C^{1}$, Martine Duclos $M^{3}$, Penfornis $A^{2}$, Pussard $E^{4}$, Not $D^{5}$, Heyman $E^{6}$, Koukoui $F^{7}$, Simon $S^{8}$, Charpentier $G^{1,2}$

${ }^{1}$ Centre for Study and Research for Improvement of the Treatment of Diabetes, Evry, France; ${ }^{2}$ Department of Diabetes, Sud-Francilien Hospital, Corbeil-Essonnes, France; ${ }^{3}$ Department of Sport Medicine and Functional Exploration, Clermont-Ferrand University Hospital, UMR1019 CRNH, Clermont-Ferrand, France; ${ }^{4}$ Molecular Genetics, Pharmacogenetics and Hormonology Laboratory, Bicêtre University Hospital, Kremlin-Bicêtre, France; ${ }^{5} R C T s$, Lyon, France; 'Physical Activity, Muscle, Health' Research Team, URePSSS, University of Lille, Lille, France; ${ }^{7}$ Department of Cardiology, Sud-Francilien Hospital, CorbeilEssonnes, France; ${ }^{8}$ Department of Endocrinology, Diabetes and Metabolic Diseases, Lyon Sud University Hospital, Pierre-Bénite, France

Diabetes Obes Metab 2015; 17: 1150-1157

\section{Background}

Aerobic exercise is known to cause hypoglycemia in T1D. Previous studies have primarily focused on the optimal doses of bolus and basal insulin for prolonged exercise in athletic patients on CSII, but the guidelines for short, exercise sessions in nonathletic patients have not been determined. This study aimed to define optimal adjustments to insulin dosing for 30 min of exercise performed $3 \mathrm{~h}$ (Part 1) or $90 \mathrm{~min}$ (Part 2) following a meal (lunch) in patients on CSII.

\section{Methods}

This was a randomized control crossover trial with 20 adult TID patients. Subjects attended multiple sessions where they exercised 3 hours postlunch; 1 rest session, 2 moderate exercise sessions (one with a $50 \%$ and the other with a $80 \%$ basal rate (BR) reduction), and 2 intense exercise sessions (one with an $80 \%$ BR reduction and the other with the pump stopped). All BR adjustments were made during exercise and remained for $2 \mathrm{~h}$ following exercise (except for the pump stopped session, where the infusion rate was re-established after exercise). Subjects returned for 2 postlunch exercises sessions that occurred 90 min postmeal in order to assess differences between adjusting bolus vs. basal insulin delivery. For all exercises, patients assessed their perceived intensity using the Borg scale.

\section{Results}

For the late exercise sessions, there were no overall differences in the occurrence of hypoglycemia with exercise versus rest. In the afternoon, there were significantly more hypoglycemic events with the milder reductions at both exercise intensities compared to rest (moderate exercise; 50\% $\mathrm{BR}$ reduction vs. rest, $P=0.03$; intense exercise, $80 \% \mathrm{BR}$ reduction vs. $=$ rest, $P=0.028$ ). For the early exercise sessions, there was a trend towards fewer hypoglycemia events with bolus versus BR reduction, but this did not reach significance $(P=0.07)$. When asked about perceived intensity, $90 \%$ of subjects accurately rated the $50 \% \mathrm{VO}_{2}$ max exercise as moderate, while the $75 \% \mathrm{VO}_{2} \max$ exercise was only accurately perceived as intense $60 \%$ of the time.

\section{Conclusions}

In adults with T1D, it is possible to exercise for $30 \mathrm{~min}, 3 \mathrm{~h}$ postlunch without increasing the risk of hypoglycemia by reducing the BR by $80 \%$ for moderate exercise and by stopping the pump altogether for intense exercise. If exercising shortly after a meal, reducing the bolus insulin dose by $50 \%$ appears to be sufficiently effective in preventing hypoglycemia.

\section{Comment}

The benefits of exercise are well known, but with fear of hypoglycemia being one of the major barriers to exercise in type 1 diabetes, there is an increased requirement to develop specific strategies to limit its likelihood of occurring. Most studies use well trained athletes, but with a significant percent of the general population being physical inactive $(\sim 36.1 \%)(12)$, a percentage that only increases in the T1D population $(\sim 63 \%)(13)$, specific guidelines for a nonathletic population are required. This study used insulin pumps as opposed to multiple daily injections and one of the major benefits to this with exercise is that adjustments can be made at the onset of 
exercise, making an employable strategy if activity cannot be foreseen in advance. This study successfully determined optimal dose adjustments of nonathletic individuals performing short bouts of exercise $(30 \mathrm{~min})$ that would be representative of daily leisure or work activity. Based on this study, an aggressive reduction of basal insulin delivery (i.e., $80 \%-100 \%$ ) might be most effective, as the $50 \%$ BR reduction did not fully prevent hypoglycemia. This adjustment was found to be in agreement with a study by McAuley and colleagues, where a $50 \%$ BR reduction was not sufficient to reduce circulating insulin levels even when it was performed an hour before the start of the activity (14).

\section{Impact of physical activity on glycemic control and prevalence of cardiovascular risk factors in adults with type 1 diabetes: a cross-sectional multicenter study of $\mathbf{1 8 , 0 2 8}$ patients}

Bohn $B^{1}$, Herbst $A^{2}$, Pfeifer $M^{3}$, Krakow $D^{4}$, Zimny $S^{5}$, Kopp $F^{6}$, Melmer $A^{7}$, Steinacker $J^{8}$, and Holl $R W^{1}$, for the DPV Initiative

${ }^{I}$ Institute of Epidemiology and Medical Biometry, ZIBMT, German Center for Diabetes Research, University of Ulm, Ulm, Germany; ${ }^{2}$ Centre for Paediatrics, Medical Clinic Leverkusen, Leverkusen, Germany, ${ }^{3}$ Diabetes Center, Clinic Tettnang, Tettnang, Germany; ${ }^{4}$ DZFO, Diabetes Centre Forchheim, Forchheim, Germany; ${ }^{5}$ Center for Internal Medicine, Endocrinology and Diabetology, Schwerin Hospital, Schwerin, Germany; ${ }^{6}$ Diabetes Center, Augsburg Clinical Center, Augsburg, Germany; ${ }^{7}$ Department of Internal Medicine I, Medical University of Innsbruck, Innsbruck, Austria; ${ }^{8}$ Division of Sports and Rehabilitation Medicine, Department of Internal Medicine II, University of Ulm, Ulm, Germany

\section{Diabetes Care 2015; 38: 1536-1543}

\section{Background}

Various studies have demonstrated the positive effects of physical activity on a multitude of diseases including cardiovascular disease, T2D and some cancers, yet studies on T1D tend to be smaller and meta-analyses have shown conflicting results for exercise recommendations. For this reason, this study aims to examine the influence of exercise on glycemic control and cardiovascular risk factors in 18,028 patients with T1D.

\section{Methods}

Subject information was provided by the Diabetes Patienten-Verlaufsdokumentation (DPV) database in Germany and Austria, which documented diabetes care strategies and their outcomes from 18,028 patients aged 18 to 80 years. Data reported included self-reported physical activity (PA), measured as frequency per week (PA2; 2 or more times per week, PA1;1 time a week, and PA0; inactive), HbA1c values, body mass index (BMI), hypertension and the use of antihypertensive drugs, dyslipidemia, diabetic ketoacidosis (DKA), and events of severe hypoglycemia and hypoglyce- mia resulting in a loss of consciousness. Data were stratified by group size and further stratified by age and gender.

\section{Results}

Frequency of PA ranged between 0 and 9 times per week. The number of subjects who reported exercising more than two times per week (PA2) was 3212 (17.8\%), 3459 subjects (19.9\%) reported to be physically active one to two times (PA1), and 11,357 (63.0\%) were inactive (PA0). Women were more often inactive compared with men (PA0: 66.0\% vs. $60.5 \%$, respectively) and the percentage of inactive individuals increased with age in both genders. PA had an inverse association with total daily insulin levels and $\mathrm{HbA} 1 \mathrm{c}$ levels, independent of age and gender $(P<0.0001)$. Additionally, physical activity was inversely associated with DKA, BMI, and dyslipidemia $(P<0.0001$ for all). Rates of severe hypoglycemia were lowest in the moderately active group. In addition to having the highest rates of hypoglycemia, the least active group had the highest incidence of retinopathy and microalbuminuria.

\section{Conclusions}

Physical activity has a beneficial effect on T1D patients in terms of improving $\mathrm{HbAlc}$ levels and associated comorbidities. Despite this, an increased percentage of subjects were reported to be inactive (63\% versus approximately $34 \%$ in the general population) and more research should focus on encouraging participation in physical activity in this patient population.

\section{Comment}

This multicenter cross-sectional study from Germany and Austria is the largest to date to demonstrate the beneficial impact of exercise on T1D. Overall, high levels of PA were associated with a lower daily insulin dose, a lower $\mathrm{HbA} 1 \mathrm{c}$ and reduced diabetes-related complications. However, with T1D, the potential for developing exercise-induced hypoglycemia is one of the greatest barriers to participating in exercise (15). In this large study, it was found that in women and in the oldest agegroup (45 to 80 years), the rate of severe hypoglycemia increased with PA, whereas in other subgroups, there was an inverse association. This finding should caution health-care providers about prescribing exercise for older women living with type 1 diabetes, whose rates of hypoglycemia are already elevated when compared to younger women (16).

\section{Do youth with type 1 diabetes exercise safely? A focus on patient practices and glycemic outcomes}

Roberts $A J^{1,2}$, Yi-Frazier $J P^{3}$, Aitken $K E^{l}$, Mitrovich $C A^{3}$, Pascual $M F^{3}$, Taplin $C E^{1,2}$

${ }^{1}$ Seattle Children's Hospital Division of Endocrinology and Diabetes, Seattle, WA; ${ }^{2}$ University of Washington, Seattle, WA; ${ }^{3}$ Seattle Children's Research Institute, Seattle, WA

Pediatr Diabetes 2016; Jul 6. [Epub ahead of print] DOI 10.1111/pedi.12402 


\section{Background}

Various insulin adjustment strategies have been tested to help reduce glycemic excursions during and after exercise, but little is known about their use in youth with type 1 diabetes. These authors aimed to assess the typical exercise management practices in youth with type 1 diabetes and examine the associations between key behaviors and glycemic control.

\section{Methods}

The "Type 1 Diabetes Report of Exercise Practices Survey (T1D-REPS)" "was developed and piloted in 100 youth with type 1 diabetes on CSII. Participants completed a 3 day physical activity recall and 30 days of pump/glucose data were collected and analysed.

\section{Results}

Eighty-four percent of participants modified their insulin regimen around exercise; only $40 \%$ reported adjusting prandial insulin immediately before exercise, while $68 \%$ reported some modification (suspension or decrease) of basal insulin during exercise. Following exercise, only $10 \%$ reported reducing basal insulin overnight. Those who performed $\geq 5$ glucose checks/day adjusted basal insulin during exercise more frequently than those with fewer daily glucose checks $(33 \%$ vs. $13 \%, P=0.05, \chi(2)=3.7)$, and were more likely to report decreasing insulin dose for the bedtime snack following exercise (50\% vs. $17 \%, P=0.004, \chi(2)=8.2$ ).

\section{Conclusions}

Basal insulin reduction at the time of exercise appears to be the most common approach to reduce the risk of exerciseassociated hypoglycemia. Despite research demonstrating that hypoglycemia after exercise is a frequent occurrence in youth, a majority $(\sim 90 \%)$ are not adjusting insulin overnight after exercise. A tool designed to capture patient practices and provide clinicians with a framework for patient education may lead to improved safety around exercise in this active patient population.

\section{Comment}

Over the last several years, a good number of clinical studies have been conducted to help inform insulin dose reductions and/or carbohydrate snacks for aerobic exercise in adolescents with type 1 diabetes $(17,18,19,20)$. Guidelines for exercise management based on these studies have been published (21) and updated (22). For youth on CSII, it should almost be automatic to reduce basal insulin infusion rates overnight by about $20 \%$ from bedtime for about 6 hours to help reduce risk for nocturnal hypoglycemia (19). Yet, according to this study, few patients, or their parents, are performing these basic adjustments. Another very recently published study demonstrates that exercise makes glucose control more difficult and that few evidenced-based practices are being used by adults with type 1 diabetes on MDI or on CSII (23). Collectively, these findings may be alarming for those of us who advocate for safe physical activity for youth with diabetes. As these authors point out, automated tools that monitor physical activity levels and then recommend basic insulin dose adjustments at bedtime are urgently needed.

\section{General practitioners' barriers to prescribe physical activity: the dark side of the cluster effects on the physical activity of their type 2 diabetes patients}

Lanhers $C^{1,5}$, Duclos $M^{1,2,5}$, Guttmann $A^{3,4}$, Coudeyre $E^{5,6}$, Pereira $B^{4,7}$, Ouchchane $L^{3,4}$

${ }^{1}$ Department of Sport Medicine and Functional Explorations, Clermont-Ferrand University Hospital, BP 68, 63001, Clermont-Ferrand, Cedex 1, France; ${ }^{2}$ Laboratory of Human Nutrition, INRA UMR 1019, Clermont-Ferrand, France; ${ }^{3}$ Clermont-Ferrand University Hospital, Department of Public Health, Biostatistics Unit, ClermontFerrand, France; ${ }^{4}$ Laboratory of Image Sciences for Interventional Techniques, UMR CNRS UdA 6284 ISIT, Auvergne University, Clermont-Ferrand, F-63001, France; ${ }^{5}$ Clermont University, University of Auvergne, ClermontFerrand, France; ${ }^{6}$ Clermont-Ferrand University Hospital, Department of Physical Medicine and Rehabilitation, Clermont-Ferrand, France; ${ }^{7}$ Clermont-Ferrand University Hospital, Innovation and Clinical Research, ClermontFerrand, France

\section{PLoS One 2015; 10: e0140429.}

\section{Background}

This research was conducted to quantify the barriers to physical activity engagement in patients with type 2 diabetes. It also determined if general practitioners (GPs) have any perceived barriers for exercise for their patients that may be impacting their activity levels.

\section{Methods}

A cross-sectional study of GPs $(n=48)$ and their type 2 diabetes patients $(n=369)$ measuring respectively barriers to prescribe and practice physical activity was conducted using a self-assessment questionnaire: barriers to physical activity in diabetes (BAPAD). Statistical analysis was performed using a hierarchical data structure. Similar practitioner's patients were assessed using a cluster sharing common patterns approach.

\section{Results}

Patients with higher barriers to physical activity were much more likely to declare practicing no physical activity $(P<0.001)$ or a lower frequency and duration of activity $(P<0.001)$. A high patient's BAPAD score was also associated with a higher risk to have a HbA1c $\geq 7 \%(53 \mathrm{mmol} /$ mol) $(P=0.001)$. The intra-class correlation coefficient between type 2 diabetes patients and GPs was $\sim 34 \%$, indicating a high cluster effect. Thus, when a GP has a high BAPAD score regarding the exercise prescription, it is predictive of a high BAPAD score with their patients, regarding their practice.

\section{Conclusions}

Overall, it appears that patients with lower BAPAD score and thus lower perceived barriers to physical activity, have 
higher physical activity levels and better glycemic control compared to those with higher BAPAD scores. A deleterious cluster effect between GPs and their patients was also found: the higher the GP's BAPAD score for their patient, the higher the patients' BAPAD score. This important cluster effect might designate GPs as a relevant lever for future interventions regarding patient's education towards exercise and type 2 diabetes management.

\section{Comment}

Physical activity is a central component of type 2 diabetes management, but a number of barriers for engagement may exist for patients. Of course, lack of time, lack of resources, poor motivation, and bad weather may all get in the way of being active if you have diabetes (or not), as may challenges with one's own diabetes control. But having your physician not think you can exercise? That is a whole other set of problems. In this study, the perceived barriers for patients with type 2 diabetes were assessed via questionnaire to 48 GPs. The survey also asked about the physicians' own engagement in regular exercise. In general, the GPs promoted regular physical activity (primarily walking) and felt that regular exercise was as important as medications for glycemic control. However, some physicians believed that their own patients were too unfit (and/or too unhealthy) to participate in exercise and that exercise would cause challenges to glucose control. For the patients, those with more barriers to exercise participation tended to have diabetes longer and tended to have higher HbA1c levels compared to those patients who had fewer barriers. Patients' BAPAD score was not linked with practitioner specialty or the physician's level of physical activity levels. But, the higher the GPs' BAPAD score, the higher the patients' BAPAD score. This finding is important, since perceived barriers decrease the likelihood of engaging in health-related behaviors that might otherwise reduce disease risks and improve patient quality of life. This research suggests that some of the barriers to physical activity engagement might be being reinforced through some of the actions of their own GPs. The next step will be to attempt to influence the GPs' perceived barriers for their patients and help them promote physical activity safely and effectively.

\section{Effects of high-intensity interval exercise versus moderate continuous exercise on glucose homeostasis and hormone response in patients with type 1 diabetes mellitus using novel ultra-long-acting insulin}

Moser $O^{1,2,3}$, Tschakert $G^{2}$, Mueller $A^{2}$, Groeschl $W^{2}$, Pieber $T^{l}$, Obermayer-Pietsch $B^{l}$, Koehler $G^{l}$, Hofmann $P^{2}$

${ }^{1}$ Department of Internal Medicine, Division of Endocrinology \& Metabolism, Medical University of Graz, Graz, Austria; ${ }^{2}$ Institute of Sports Sciences, Exercise Physiology \& Training Research Group, University of Graz, Graz, Austria; ${ }^{3}$ Center of Sports Medicine \& Sports Orthopedics, University Outpatient Clinic, University of Potsdam, Potsdam, Germany

PLoS One 2015; 10: e0136489

This manuscript is discussed also in Article on New Insulin, Biosimilars, and Insulin Therapy, page S-42.

\section{Background}

Hypoglycemia during exercise can be ameliorated in MDI patients with type 1 diabetes by reducing the prandial insulin dose at the meal before exercise. Post-exercise hypoglycemia can also be managed with this approach by reducing the bolus insulin at the meal after exercise. The purpose of this study was to determine the efficacy of reducing the meal time boluses by $25 \%, 50 \%$, and $75 \%$ for continuous $(\mathrm{CON})$ and intermittent high intensity interval exercise (HIIE) at low, moderate, and high workloads in patients with type 1 diabetes on MDI.

\section{Methods}

Seven males with type 1 diabetes, all using insulin degludec (Tresiba/Novo Nordisk, Denmark) as basal insulin, performed either HIIE or CON exercise at one of three different intensities (low, moderate, high) on a cycle ergometer. Before exercise, standardized meals were administered, and short-acting insulin dose was reduced by $25 \%$ (low intensity trial), $50 \%$ (moderate intensity trial), or $75 \%$ (high intensity trial).

\section{Results}

Blood glucose (BG) decrease during HIIE was significantly smaller for the moderate intensity exercise $(P=0.024)$ and tended to be smaller for the other intensities compared to the CON trials. No differences were found for post-exercise interstitial glucose, acute hormone response, and carbohydrate utilization between HIIE and CON for low, moderate, and high intensity exercise. In HIIE, blood lactate for the low intensity $(P=0.006)$ and moderate intensity $(P=0.004)$, as well as respiratory exchange ratio for low $(P=0.003)$ and moderate intensity ( $P=0.003)$ were significantly higher compared to $\mathrm{CON}$ but not for the high intensity exercise.

\section{Conclusions}

Hypoglycemia did not occur during or after HIIE and CON when using ultra-long-acting insulin and when applying a bolus dose insulin reduction for exercise. In general, HIIE tended to be associated with a lesser reduction in glycemia when compared to $\mathrm{CON}$ exercise, even with markedly higher peak workloads occurring with the former type of exercise.

\section{Comment}

People with type 1 diabetes perform all sorts of different types of exercise. In this study, it was shown that activities that have brief periods of higher intensity effort, called high intensity interval exercise (HIIE) tend to be associated with less risk for hypoglycaemia compared with continuous exercise. This study also shows that, in general, higher intensity aerobic exercise performed for 30 min requires greater reductions in prandial insulin at the meals before and after exercise than milder forms 


\section{Results}

Bed-rest resulted in $29 \% \pm 5 \%$ decrease in insulin resistance $(P<0.01)$. Additionally, a decrease was observed in lean tissue (i.e., muscle and bone) mass $1.4 \pm 0.2 \mathrm{~kg}$ $(P<0.01)$, accompanied with a $3.2 \% \pm 0.9 \%$ decline in quadriceps cross-sectional area $(P<0.01)$. Impairments in peak oxygen uptake $\left(\mathrm{VO}_{2}\right.$ peak) were also observed, decreasing by $6.4 \% \pm 2.3 \%(P<0.05)$, while one repetition $\max (1 \mathrm{RM})$ was reduced $6.9 \% \pm 1.4 \%(P<0.01)$. Lipid content, mitochondrial function and capillary density were unchanged in muscle biopsy samples.

\section{Conclusions}

Overall, bed rest resulted in significant impairments to metabolic health and the mechanisms for the impairments in glucose tolerance were not associated with those that are typically associated with high-fat diet induced insulin resistance.

\section{Comment}

The benefits of exercise on improving overall glycemic control seem to be well understood (28), but this study takes another angle and highlights the detrimental implications of impaired physical activity in the extreme setting of bed rest. A report in 2014 revealed that $\sim 25 \%$ of all Americans were not getting any form of leisure physical activity (29). In this study, it is highlighted that after just one week of bed rest, there is a significant detriment in insulin sensitivity, reducing glucose disposal by about $30 \%$. Additionally, in the one week trial, the body went through physiological changes that would take 12 weeks of intensive exercise to revert. It was surprising to find was that the expected mechanisms for the impaired insulin sensitivity could not account for the changes they observed. Increases in insulin resistance occurred before changes in key muscle proteins known to regulate glucose uptake, or before any detected changes in lipid profiles or accumulation, or before dysfunction of mitochondria, or reduced capillary density. One potential mechanism may simply be the loss of muscle mass, resulting in a reduced capacity for glucose uptake. Although the precise mechanism remains to be elucidated, this study emphasizes the detrimental effects of inactivity. Future strategies should focus on strategies to limit periods of immobility, specifically with hospitalization, as well as creating programs to encourage physical activity as a proactive treatment to help glycemic control.

\section{Author Disclosure Statement}

M.C.R. has been an industry sponsored speaker for Medtronic, Sanofi, Ascencia Diabetes Care, and Eli Lilly. A.M.P. has no competing financial interests.

\section{References}

1. Marliss EB, Vranic M. Intense exercise has unique effects on both insulin release and its roles in glucoregulation: implications for diabetes. Diabetes 2002; 51 Suppl 1: S271-83.

2. Yardley JE, Sigal RJ, Perkins BA, Riddell MC, Kenny GP. Resistance exercise in type 1 diabetes. Can $J$ Diabetes 2013; 37: 420-26.

3. Yardley JE, Iscoe KE, Sigal RJ, Kenny GP, Perkins BA, Riddell MC. Insulin pump therapy is associated with less post-exercise hyperglycemia than multiple daily injections: an observational study of physically active type 1 diabetes patients. Diabetes Technol Ther 2013; 15: 84-88.

4. Benbenek-Klupa T, Matejko B, Klupa T. Metabolic control in type 1 diabetes patients practicing combat sports: at least two-year follow-up study. SpringerPlus 2015; 4: 133.

5. Fahey AJ, Paramalingam N, Davey RJ, Davis EA, Jones TW, Fournier PA. The effect of a short sprint on postexercise whole-body glucose production and utilization rates in individuals with type 1 diabetes mellitus. J Clin Endocrinol Metab 2012; 97: 4193-200.

6. Davidson PC, Hebblewhite HR, Steed RD, Bode BW. Analysis of guidelines for basal-bolus insulin dosing: basal insulin, correction factor, and carbohydrate-to-insulin ratio. Endocr Pract 2008; 14: 1095-101.

7. Jacobs PG, Resalat N, El Youssef J, Reddy R, Branigan D, Preiser N, Condon J, Castle J. Incorporating an Exercise Detection, Grading, and Hormone Dosing Algorithm Into the Artificial Pancreas Using Accelerometry and Heart Rate. J Diabetes Sci Technol 2015; 9: 1175-184.

8. Das P, Biswas S, Mukherjee S, Bandyopadhyay SK. Association of oxidative stress and obesity with insulin resistance in type 2 diabetes mellitus. Mymensingh Med $\mathrm{J}$ 2016; 25: 148-52.

9. Jiménez-Pavón D, Ruiz JR, Ortega FB, Martínez-Gómez D, Moreno S, Urzanqui A, Gottrand F, Molnár D, Castillo MJ, Sjöström M, Moreno LA: HELENA Study group. Physical activity and markers of insulin resistance in adolescents: role of cardiorespiratory fitness levels-the HELENA study. Pediatr Diabetes 2013; 14: 249-58.

10. Shalev-Goldman E, McGuire KA, Ross R. Waist circumference and cardiorespiratory fitness are independently associated with glucose tolerance and insulin resistance in obese women. Appl Physiol Nutr Metab 2014; 39: 358-62.

11. Ross R, de Lannoy L, Stotz PJ. Separate effects of intensity and amount of exercise on interindividual cardiorespiratory fitness response. Mayo Clin Proc 2015; 90: 1506-514.

12. Oggioni C, Lara J, Wells JCK, Soroka K, Siervo M. Shifts in population dietary patterns and physical inactivity as determinants of global trends in the prevalence of diabetes: An ecological analysis. Nutr Metab Cardiovasc Dis 2014; 24: 1105-111.

13. Bohn B, Herbst A, Pfeifer M. Krakow D, Zimny S, Kopp F, Melmer A, Steinacker JM, Holl RW, DPV Initiative. Impact of physical activity on glycemic control and prevalence of cardiovascular risk factors in adults with type 1 diabetes: A cross-sectional multicenter study of 18,028 patients. Diabetes Care 2015; 38: 1536-543.

14. McAuley SA, Horsburgh JC, Ward GM, La Gerche A, Gooley JL, Jenkins AJ, MacIsaac RJ, O’Neal DN. Insulin pump basal adjustment for exercise in type 1 diabetes: a randomised crossover study. Diabetologia 2016; 59: 1636644.

15. Brazeau A-S, Rabasa-Lhoret, R, Strychar I, Mircescu H. Barriers to physical activity among patients with type 1 diabetes. Diabetes Care 2008; 31: 2108-109. 
16. Miller KM, Foster NC, Beck RW, Bergenstal RM, DuBose SN, DiMeglio LA, Maahs DM, Tamborlane WV, T1D Exchange Clinic Network. Current state of type 1 diabetes treatment in the U.S.: updated data from the T1D Exchange clinic registry. Diabetes Care 2015; 38: 971-78.

17. Riddell MC, Milliken J. Preventing exercise-induced hypoglycemia in type 1 diabetes using real-time continuous glucose monitoring and a new carbohydrate intake algorithm: an observational field study. Diabetes Technol Ther 2011; 13: 819-25.

18. Riddell MC, Bar-Or O, Ayub BV, Calvert RE, Heigenhauser GJ. Glucose ingestion matched with total carbohydrate utilization attenuates hypoglycemia during exercise in adolescents with IDDM. Int J Sport Nutr 1999; 9: 24-34.

19. Taplin CE, Cobry E, Messer L, McFann K. Chase HP, Fiallo-Scharer R. Preventing post-exercise nocturnal hypoglycemia in children with type 1 diabetes. J Pediatr 2010; 157: 784-88.e1.

20. The Diabetes Research in Children Network (DirecNet) Study Group. Prevention of hypoglycemia during exercise in children with type 1 diabetes by suspending basal insulin. Diabetes Care 2006; 29: 2200-204.

21. Robertson K, Riddell MC, Guinhouya BC, Adolfsson P, Hanas R. Exercise in children and adolescents with diabetes. Pediatr Diabetes 2014; 15 Suppl 20: 203-23.

22. Pivovarov JA, Taplin CE, Riddell MC. Current perspectives on physical activity and exercise for youth with diabetes. Pediatr Diabetes 2015; 16: 242-55.

23. Pinsker JE, Kraus A, Gianferante D, Schoenberg B. E. Singh SK, Ortiz, H, Dassau E, Kerr D. Techniques for exercise preparation and management in adults with type 1 diabetes. Can J Diabetes. 2016. Article in Press DOI: http://dx.doi.org/10.1016/j.jcjd.2016.04.010

24. Shetty VB, Fournier PA, Davey RJ, Retterath AJ, Paramalingam N, Roby HC, Cooper MN, Davis EA, Jones TW. Effect of exercise intensity on glucose requirements to maintain euglycemia during exercise in type 1 diabetes. $J$ Clin Endocrinol Metab 2016; 101: 972-80.

25. Cinar A, Turksoy K, Quinn L, Littlejohn E. An integrated hypoglycemia early alarm and adaptive control system for artificial pancreas. Diabetes Technol Ther 2014; 16 [Suppl 1]: A103-A103.

26. Farabi SS, Carley DW, Cinar A, Quinn L. Routine daily physical activity and glucose variations are strongly coupled in adults with T1DM. Physiol Rep 2015; 3: e12644

27. Jacobs PG, Resalat N, El Youssef J, Reddy R, Branigan D, Preiser N, Condon J, Castle J. Incorporating an exercise detection, grading, and hormone dosing algorithm into the artificial pancreas using accelerometry and heart rate. J Diabetes Sci Technol 2015; 9: 1175-184.

28. Tomas-Carus P, Ortega-Alonso A, Pietilainen KH, Santos V, Goncalves H, Ramos J, Raimundo A. A randomized controlled trial on the effects of combined aerobicresistance exercise on muscle strength and fatigue, glycemic control and health-related quality of life of type 2 diabetes patients. J Sports Med Phys Fitness 2016; 56: 572-78.

29. McGuire S. Centers for Disease Control and Prevention. State Indicator Report on Physical Activity, 2014. Atlanta GA: US Department of Health and Human Services, 2014. Adv Nutr. 5(6): 762-63. 